cases not recorded, quinine in 5-10-grain doses and sodium salicylate in 30-grain doses were given three times a day. Further observation is necessary before the effect of these drugs can be definitely stated, but neither appeared to influence the temperature to any appreciable extent. For the relief of the pain salicylates and massage are undoubtedly useful. The diet was not necessarily restricted, and even during the fever, unless the temperature was very high, meat was given without ill effect.

Until more is known of the etiology little can be said about prophylaxis. The question of its contagiousness is an important one; it is certain that it is not contagious in any high degree, for, although no precautions for isolation were taken, no patients in the same ward developed the disease. One orderly in the ward, however, had a typical attack with one relapse.

\section{BACTERIAL ANTAGONISM, WITH PARTICULAR REFERENCE TO MENINGOCOCCUS.}

By LEONARD COLEBROOK, M.B., B.S. LoND., ASSISTANT, INOCULATION DEPARTMENT, ST. MARY'S HOSPITAL.

(From the Inooulation Department, St. Mary's Hospital.)

IN the course of an investigation of meningococcus carriers, undertaken on behalf of the Medical Research Committee, I noticed on several occasions that when the meningococcus began to disappear from the naso-pharynx it was at once replaced by great numbers of pneumococci or streptococci. Moreover, on at least two occasions the disappearance of the meningococcus seemed to be determined by the onset of an acute "cold in the head." The interesting question arose-Had these pneumococci and streptococci simply entered on a field vacated by the meningococcus, or had they also played an active rôle in supplanting that invader?

With a view of showing if there was any antagonism between these organisms on artificial media they were planted, at the suggestion of Captain S. R. Douglas, I.M.S. (ret.), in the following manner. A drop of pneumococcus culture in broth was allowed to trickle across the middle of a serumagar plate, and as soon as the track of this stream had dried in the incubator a drop of meningococcus culture was run across the plate at right angles to the pneumococcus stream. After incubation of the plate overnight it was found that where the two streams crossed the meningococcus culture had been completely inhibited by the growth of pneumococcus, and this notwithstanding that the meningococcus grew more rapidly. (Fig. 1.)

From further experiments of this kind it appeared that a similar antagonism to meningococcus is shown by many different varieties of streptococcus obtained from the normal mouth and naso-pharynx; and also by several strains of the streptococcus fæcalis type cultivated from war wounds. Moreover, it was pointed out to me by Major M. H. Gordon, R.A.M.C. (T.), that saliva (contaminated, as it always is, by very many streptococci) exercises a similar check upon the growth of meningococcus on artificial media. On the other hand, it may be noted in passing that meningococcus is not alone in its susceptibility to this inhibitory influence. With the exception of gonococcus (two strains) and micrococcus melitensis (one strain) all the
Gram-negative cocci I have so far tried have been inhibited by the test culture of pneumococcus employed. They comprise some three or four strains of M. catarrhalis from the nose and mouth, and several strains of the ill-classified cocci which somewhat closely resemble meningococcus, yet differ from that organism by their sugar reactions, or the colour of colonies, or their ability to grow readily at $23^{\circ} \mathrm{C}$. I have failed to find any evidence of a reciprocal influence exerted by the meningo. coccus upon the pneumococci or streptococci antagonistic to it.

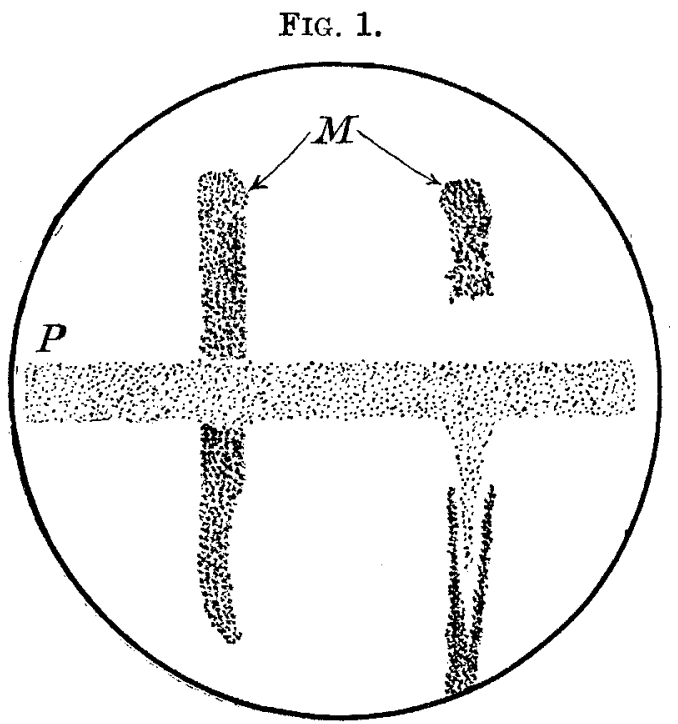

Plate culture showing checking of meningococcus (vertical streaks) by pneumococcus (horizontal). The meningococcus stream on the left was planted from above downwards a few minutes after the pneumococcus - that on the right several hours later, to show checking at a distance. It will be noticed that pneumococci have been carried down in this stream. $M=$ meningococcus. $P=$ pneumococcus.

This last observation raises a point of some prac. tical importance-viz., that as the antagonism is one-sided, it may evidently operate to conceal the presence of meningococi in cultures made from the post-nasal swabs of meningitis contacts-particularly if a relatively small number of meningococci happen to be mixed with a larger "catch" of inhibitory pneumococci or streptococci, picked up by the swab during its withdrawal through the mouth, or from the naso-pharynx. If, however, the meningococcus predominates on the swab its growth will not be checked-a point which will be brought out a little later.

The following experiments were directed chiefly to determining the nature of this antagonism. Although they have been performed usually with one particular strain of pneumococcus, it seems likely that the inferences drawn from them will be found applicable to the other meningococcusinhibiting organisms. On the plate culture represented in Fig. 1 it will be seen that by allowing the growth of pneumococcus to get well started before the meningococcus stream was planted the growth of the latter was totally checked, not only where the two streams met, but also to a distance of nearly a centimetre on either side of that point. This checking at a distance suggests at once that some change has been produced in the medium by the growth of pneumococcus. To establish this point it was necessary to eliminate the presence of the inhibitory microbes on the surface of the medium. A slope culture of pneumococcus was washed off with sterile broth and then heated at $54^{\circ} \mathrm{C}$. for an hour to kill any remaining pneumococci. Meningococci planted afterwards on this medium were unable to grow. On another occasion a serum-agar slope was heavily planted with 
pneumococci but kept at room temperature. After 20 hours the cocci were washed off and the tube heated at $54^{\circ} \mathrm{C}$. Subsequent planting with meningo. coccus gave the usual rapid and copious growth, showing that the inhibitory change in the medium is not produced by the mere presence of the pneumococcus on its surface, but only by the process of its active growth.

By another simple crosswise planting experiment -shown in Fig. 2-a further clue to the exact nature of the antagonism was obtained. The pneumococcus stream in this case was crossed by a thin and a

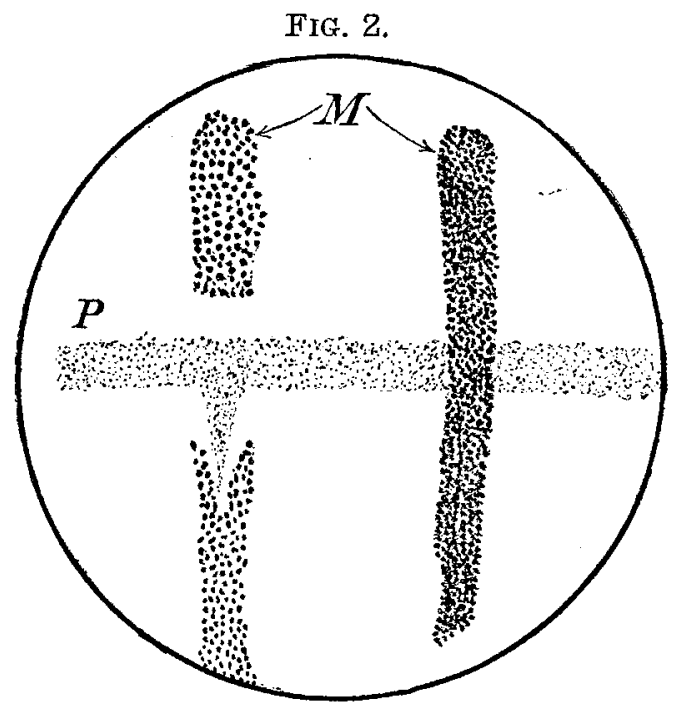

Plate culture showing the inhibition of a small number of meningococci by a pneumococcus growth which quite failed to inhibit a much larger number. The meningococcus streams were planted about 15 minutes after the pneumo-
coccus. $M=$ meningococcus. $P=$ pneumococcus.

thick sowing, respectively, of meningococci, with the rather unexpected result that the many microbes of the thick sowing were able to produce a vigorous growth across the area forbidden to the few. Clearly, then, the pneumococci had not deprived the medium of something which was essential for the growth of meningococcus, nor in any other way had they rendered the medium, as such, unsuitable for that organism.

The suggestion remains that the pneumococcus by its growth diffuses into the surrounding medium some substance which is directly bactericidal to meningococcus. Such a bactericidal process might well be adequate to check (by death) only a certain limited number of meningococci, as shown in Fig. 2; and, moreover, it might be expected to produce the sharp line of demarcation between inhibited and uninhibited meningococci which is a rather striking feature of these crosswise planting experiments (vide Figs. 1 and 2).

Direct evidence of such a poisoning or bactericidal process was obtained by working with fluid cultures of pneumococcus. A well-grown culture in glucose-ascitic-broth was sterilised by heat, or filtered, and then inoculated with a certain number of meningococci. By testing samples of this for sterility at appropriate intervals it was found that, unless too large a number had been implanted, the meningococci were completely killed off within a short period of exposure to the pneumococcuspoisoned broth. From an average of several quantitative experiments it appeared that at least 40,000 cocci were thus killed within an hour by 1 c.c. of the pneumococcus broth. The details of the experiment were as follows:-

Broth suspensions of convenient strength were prepared from a very young meningococcus culture; and the living cocci in them enumerated by spreading small sample volumes -after appropriate dilution-upon agar surfaces, and counting the colonies which resulted. $10 \mathrm{c.mm}$. volumes of these broth suspensions of meningococci were mixed with $\frac{1}{2}$ c.c. volumes of a glucose-ascitic-broth culture of pneumococcus previously sterilised by heating at $54^{\circ} \mathrm{C}$. for an hour, or a sterile filtrate of such a culture. After incubation for half an hour or an hour, ${ }^{1} 10 \mathrm{c} . \mathrm{mm}$. volumes were withdrawn from each of the tubes and tested for sterility by planting in 10 c.c. of ascitic broth. This quantity10 c.c. - was chosen in order to dilute 1000 -fold any inhibitory substance which might be carried over in the $10 \mathrm{c.mm}$. volumes to be tested, such a dilution having been previously found more than adequate to render the inhibitory substance in fluid cultures ineffective. These 10 c.c. test volumes of ascitic broth were contained in small flat-bottomed flasks in preference to test-tubes for the better aeration of the medium -a point of some importance in making fluid cultures from a small sowing of meningococci.

As was stated summarily above, the usual result of these tests was that the $10 \mathrm{c} . \mathrm{mm}$. samples carried over no living meningococci into the ascitic broths, which remained sterile except in the case when more than 30,000 or 40,000 cocci had been originally introduced into the $\frac{1}{2}$ c.c. volumes of pneumococcus culture. By way of control to this experiment it was necessary to show that the medium (glucose-asciticbroth) which had, apparently, been rendered bactericidal to meningococcus by the previous growth of pneumococcus in it, was not itself contributory to their rapid death. For that purpose samples of the broth were inoculated with meningococci and incubated for an hour precisely' as in the case of the heated pneumococcus culture, but instead of being simply tested for sterility after the period of incubation it was submitted to a count of its living cocci, both before and after that period, by the method described above for enumerating the meningococcal suspensions. The result of these control experiments showed-as was to be expected - a slight increase, rather than any diminution, of the living organisms within the period of incubation.

Little is yet known in regard to this bactericidal process. That it is not dependent on acid formation by the pneumococcus is shown by the fact that on medium free from sugar that microbe produces no acid and yet inhibits meningococcus strongly. Nor is it dependent on the presence of serum or serous fluid in the medium. All that can be positively stated at present is that the bactericidal agent, whatever it may be, is filterable, and is inactivated at a temperature of between $70^{\circ}$ and $80^{\circ} \mathrm{C}$. I have been unable to find any evidence that it exerts a lytic effect upon the meningococcus.

Antagonism among bacteria has been recognised for many years. Kitasato ${ }^{2}$ in 1889 drew attention to the fact that several microbic species are prevented from growing, and even killed, by the contiguous growth of the cholera vibrio, while that microbe in turn is inhibited by the bacillus pyocyaneus. Later it was shown that the bacillus pyocyaneus is also -and in greater degree-antagonistic to the diphtheria bacillus. In 1903 Lode $^{3}$ published a descrip. tion of a certain large coccus isolated from the air which exerts a strong inhibitory influence upon the growth of such diverse organisms as the bacilli of anthrax and chicken cholera, the staphylococcus, and the micrococcus tetragenus; and also a somewhat less potent effect upon the bacillus typhosus and the cholera vibrio.

With two of these organisms- - viz., micrococcus tetragenus and the bacillus of chicken cholera-he was able to show that the inhibition was dependent upon a definite bactericidal action exerted by the medium in which the coccus had grown-the

1 A longer exposure to the action of the pneumococcus culture would have rendered the result fallacious owing to the notable tendency of meningococcus to die very rapidly in any medium which is not actually favourable to its growth.

3 Lode: 2 Kitasato: Zeitschrift für Hygiene, 1889.

3 Lode : Centralblatt für Bakteriologie, Orig., Band xxxiii. 
bactericidal agent being a filterable and dialysable substance inactivated at a temperature of $97^{\circ} \mathrm{C}$.

It is in connexion with its power to check the growth of the cholera vibrio, however, that this coccus has chiefly claimed attention. Metchnikoff ${ }^{4}$ has long entertained the idea that individuals who escape cholera, when that disease is rife in a community, may owe their protection to the presence in the intestinal canal of these air-borne cocci or of other organisms, like bacillus pyocyaneus, antagonistic to the development of the vibrios. This suggestion was recently taken up by Choukevitch, ${ }^{5}$ who infected with cholera vibrios several rabbits which had previously ingested large numbers of the "anti-cholera" cocci with their food. Although the rabbits developed cholera and died like the control animals no inference could be drawn from the experiment, since postmortem examinations did not indicate that the "anti-cholera" cocci had been able to survive in large numbers and maintain a vigorous growth in the intestinal canal.

Whether or not the future will establish this conception with regard to cholera it would seem probable that the principle of bacterial antagonism will be found to have other, and perhaps therapeutically useful, applications in the wide field of microbic infections. Two examples readily suggest themselves in connexion with meningococcus infections. First, it may well be that the presence of meningococcus-inhibiting pneumococci or streptococci on the nasal and pharyngeal mucous membranes will determine the fate of a small number of meningococci deposited there from the inspired air. And secondly, the observations recorded in the first paragraph of this paper suggest that the operation of antagonism may sometimes effect the "cure" of meningococcus carriers.

From this the question arose whether one could bring about a similar "curative" procedure at will. The application of a filtrate of pneumococcus culture, however strongly bactericidal to meningococcus, could not be expected to give any better success than the use of antiseptics, which apparently fail of their object because they do not gain access to all the meningococci. It therefore seemed necessary to implant a living culture of the inhibitory microbes upon the naso-pharynx, in the hope that these would be able to establish themselves and spread over all the area invaded by the meningococci. The pneumococcus culture with which most of the inhibition experiments had been performed had come from a healthy ex-meningococcus carrier, with no history of pneumonia, and had, since its isolation, been passed through 20 or 30 generations on artificial media.

These considerations, and the fact that the pneumococcus is usually present in the normal respiratory passages, led me to regard its use as quite devoid of danger. Broth cultures of it were sprayed first on the naso-pharynx of my fellow worker, Dr. Harold Tanner, whose help in this part of the inquiry I wish here to acknowledge, and subsequently on five carriers, with and without preliminary sterilisation of the naso-pharyngeal surface by silver iodide. To our surprise we found that the pneumococci were apparently unable to establish themselves in the naso-pharynx of these carriers, for although implanted in very large numbers they could not be recovered in any quantity from swabs taken after a few hours or on the following day. In the case of another carrier we implanted streptococci grown from his own saliva, but these also seemed unable to gain any hold in the naso-pharynx. Apart from their "taking root" and multiplying in the naso-pharynx, it was not to be expected that the pneumococci or streptococci introduced would have any inhibitory effect upon the meningococcus established there, and in five of the six carriers no evidence of such an effect was noticed. In the sixth case the pneumococci probably gained a slight hold at first, for the meningococci almost disappeared from swabs for nearly two days, but after that time regained their earlier pre. dominance. This last partial success seems, at any rate, to suggest that, with more knowledge of the precise conditions which enable the inhibitory organisms to establish themselves, the method might prove of some value.

My thanks are due to Captain S. R. Douglas for helpful criticism and counsel in connexion with this work.

St. Mary's Hospital, W.

\section{BLACKWATER FEVER.}

BY R. W. BURKITT, F.R.C.S. IREL.

THE following, which is a report to the principal medical officer of British East Africa on my findings and treatment in blackwater, may be of interest.

The patient, an intelligent European aged 53, had blackwater some four or five months ago, for which I attended him. For the last four months he had been living in a malarial district and had been having a lot of fever. On Sept. 12th he took 20 grains of quinine hydrochloride; this brought on blackwater in a few hours. With it came vomiting, diarrhoa, and jaundice. He knew in a common-sense way how to treat himself before my seeing him. He was brought into Nairobi on the morning of Sept. 14th, some 40 miles in a motor-car. He brought in with him his urine of the 13th. The urine of the $13 \mathrm{th}$ and the $14 \mathrm{th}$ contained both oxy- and met-hæmoglobin.

I had been noticing for the last year that all blackwater urines are excessively acid. This man's urine of the 13th and the 14th contained an enormous quantity of acetone, and showed the aceto-acetic acid reaction very markedly. These samples were highly acid by litmus paper. His kidneys are quite sound.

At midday on the 14th, as soon as I found the acetone, I began giving potassium bicarbonate $3 \mathbf{i}$. every hour in heaps of water; he was able to keep down six doses. I then lessened the dose and gave sodium bicarbonate, with the result that he passed urine very freely, and next morning his urine was strongly alkaline, with not a trace of hæmoglobin, but still with a small quantity of acetone. He was feeling much better, his jaundice was clearing, he was passing large quantities of urine, his spleen was less tender, and his diarrhoa was almost gone (this went pari passu with the acetone). On the 16th there was no hæmoglobin and no acetone $;$ he was much better and was hungry. The urine was decidedly alkaline. (Note that the bæmoglobin disappeared 24 hours before the last trace of acetonuria.)

It will be seen that $\mathrm{I}$ gave $\mathrm{CaCl}_{2}$ as a set-off to potassium and sodium-they all three are blood alkalinisers. As soon as acetone was gone I put him on increasing doses of quinine. When last I treated this man for an attack of blackwater fever $\mathrm{CaCl}_{2}$ (which is an alkaliniser, but not nearly so strong as potassium) apparently prevented hæmoglobinuria from recurring.

His history was as follows: I found him suffering from fever and very ill (he had been having fever for months). I gave him an injection of quinine gr. xx. at $10 \mathrm{~A}$.M. and told him to drink plenty of water. At 5 P.M. he was passing urine as black as ink and was very ill indeed. I gave him $\mathrm{CaCl}_{2} 3 \mathrm{i}$., which he kept down. Next morning the blackwater was gone. I then gave an injection of quinine gr. $x: x v$., and he took $\mathrm{CaCl}_{2}$ gr. $\mathrm{xx}$. every two hours for four times. He drank large quantities of water, tea, and coffee. With this larger dose of quinine he showed no sign of blackwater. 